\title{
Disorder-induced quantum phase transitions in three-dimensional second-order topological insulators
}

\author{
C. Wang $\odot^{1, *}$ and X. R. Wang $\odot^{2,3, \uparrow}$ \\ ${ }^{1}$ Department of Physics, Center for Joint Quantum Studies, School of Science, Tianjin University, Tianjin 300350, China \\ ${ }^{2}$ Department of Physics, The Hong Kong University of Science and Technology (HKUST), Clear Water Bay, Kowloon, Hong Kong \\ ${ }^{3}$ HKUST Shenzhen Research Institute, Shenzhen 518057, China
}

(Received 30 May 2020; accepted 1 September 2020; published 30 September 2020)

\begin{abstract}
Disorder effects on three-dimensional second-order topological insulators (3DSOTIs) are investigated numerically and analytically. The study is based on a tight-binding Hamiltonian for noninteracting electrons on a cubic lattice with a reflection symmetry that supports a 3DSOTI in the absence of disorder. Interestingly, unlike the disorder effects on a topological trivial system that can only be either a diffusive metal (DM) or an Anderson insulator (AI), disorders can sequentially induce four phases of 3DSOTIs, three-dimensional first-order topological insulators (3DFOTIs), DMs, and AIs. At a weak disorder when the on-site random potential of strength $W$ is below a low critical value $W_{c 1}$ at which the gap of surface states closes while the bulk sates are still gapped, the system is a disordered 3DSOTI characterized by a constant density of states and a quantized integer conductance of $e^{2} / h$ through its chiral hinge states. The gap of the bulk states closes at a higher critical disorder $W_{c 2}$, and the system is a disordered 3DFOTI in a lower intermediate disorder between $W_{c 1}$ and $W_{c 2}$ in which electron conduction is through the topological surface states. The system becomes a DM in a higher intermediate disorder between $W_{c 2}$ and $W_{c 3}$ above which the states at the Fermi level are localized. It undergoes a normal three-dimensional metal-to-insulator transition at $W_{c 3}$ and becomes the conventional AI for $W>W_{c 3}$. The self-consistent Born approximation allows one to see how the density of bulk states and the Dirac mass are modified by the on-site disorders.
\end{abstract}

DOI: 10.1103/PhysRevResearch.2.033521

\section{INTRODUCTION}

Topological states of matter have attracted much attention in condensed-matter physics in recent years because of their exotic properties, such as the topologically protected surface and edge states. These states can exist in both topological insulators [1-5] and Weyl semimetals [6]. Their existences are guaranteed by the bulk-boundary correspondence rooted in the Stokes-Cartan theorem. Fermions or bosons and classical or quantum particles, such as electrons [4,5], phonons [7-11], photons [12-14], and magnons [15-18], can have topological states. According to the bulk-boundary correspondence, a three-dimensional (3D) insulator with band inversion [5] has topologically nontrivial two-dimensional surface states. This insulator is a 3D first-order topological insulator (3DFOTI). When the surface states of a 3DFOTI are gapped, the intersection of two surfaces of different topological classes, i.e., a hinge, has topologically nontrivial chiral

\footnotetext{
*Corresponding author: physcwang@tju.edu.cn

${ }^{\dagger}$ Corresponding author: phxwan@ust.hk

Published by the American Physical Society under the terms of the Creative Commons Attribution 4.0 International license. Further distribution of this work must maintain attribution to the author(s) and the published article's title, journal citation, and DOI.
}

hinge states, leading to a so-called 3D second-order topological insulator (3DSOTI). The well-accepted paradigm is that a $d$-dimensional material can be a $(d-n)$ th-order topological insulator with $1 \leqslant n \leqslant d$ so that all states in submanifolds, whose dimensions are greater than $(d-n+1)$, are gapped whereas states are gapless on, at least, one submanifold of dimension $(d-n)$ [19-27].

All those newly discovered genuine topological phases should survive in disorders that have profound effects on electronic structures as demonstrated in the topological Anderson insulators [28-30]. The second-order topological insulators, characterized by in-gap topological states in the $(d-2)$ dimensional boundary, are the current focus in the field because of its experimental realizations in phononics [9-11], photonics [14], and circuitry [31]. So far, most of the works are on the constructions of second-order topological insulators in crystals with well-defined crystalline symmetries, the fate of such a phase under disorder has been less studied [32-35]. Thus, it should be very interesting to find out how the hinge states in 3DSOTIs are modified by disorders. From the knowledge of Anderson localization for topologically trivial states, it is known that the competition between the energy randomness and kinetic energy (the bandwidth) determines the metal-to-insulator transition. For 3DSOTIs, the gap of bulk states $\Delta_{1}$ and the gap of topological surface states $\Delta_{2}<$ $\Delta_{1}$ should be important because a transition from topological surface states to topological states on hinges, boundaries of surfaces, can only happen when the surface-state gap closes 
at the transition point. We expect that a 3DSOTI undergoes three phase transitions involving four phases as disorder strength $W$ increases. The 3DSOTI remains stable up to a critical disorder $W_{c 1}$ at which the gap $\Delta_{2}$ of surface states closes $\left(\Delta_{2}=0\right)$ whereas bulk gap remains open $\left(\Delta_{1} \neq 0\right)$. The system enters into the 3DFOTI from the 3DSOTI. Aurther increase in disorder to the second critical value of $W_{c 2}$ at which bulk gap closes, the 3DFOTI is replaced by the conventional topologically trivial diffusive metal (DM). The third quantum phase transition is expected to occur at a strong critical disorder $W_{c 3}$ at which all states are localized and the system becomes an Anderson insulator (AI). The route of these quantum phase transitions is presented schematically in Fig. 1.

In this paper, we use a 3DSOTI model of class A with a reflection symmetry in the ten Altland-Zirnbauer classification [36] to verify the generic route depicted in Fig. 1. Using highly accurate numerical calculations, we show that above three quantum phase transitions occur, indeed, when disorder strength $W$ increases. The 3DSOTI is featured by the quantized Hall conductance exactly at $e^{2} / h$ and constant density of states. The 3DFOTI is featured by its dominated occupation probability on surfaces and negligible occupation probabilities in the bulk and on hinges. The DMs and AIs are identified from the scaling analysis of the participation ratios (PRs), defined as $p_{2}(E, W)=\left\langle\left(\sum_{i}\left|\psi_{i}(E)\right|^{4}\right)^{-1}\right\rangle$ with $\left|\psi_{i}(E)\right|$ being the normalized wave-function amplitude at site $i$. The convincing numerical results are also confirmed by the self-consistent Born approximation (SCBA) calculations.

This paper is organized as follows. The tight-binding model of 3DSOTI is introduced in Sec. II. Section III demonstrates the existence of chiral hinge states in the clean limit. Various numerical results are given in Sec. IV to demonstrate the route of quantum phase transitions in Fig. 1 for a specific set of parameters. In Sec. V, a general phase diagram in the plane of the Dirac mass $M$ and the disorder strength $W$ is given, followed by the conclusion in Sec. VI.

\section{TIGHT-BINDING MODEL}

Our model is noninteracting electrons on a cubic lattice of lattice constant $a=1$ [20],

$$
\begin{aligned}
H= & \sum_{i} c_{i}^{\dagger}\left(v_{i} \Gamma^{0}+M \Gamma^{2}+B \Gamma^{31}\right) c_{i} \\
& +\left(\frac{t}{2} \sum_{\langle i j\rangle} c_{i}^{\dagger} \Gamma^{2} c_{j}+\frac{i t}{2} \sum_{i}\left(c_{i+\hat{x}}^{\dagger} \Gamma^{4}\right.\right. \\
& \left.\left.+c_{i+\hat{y}}^{\dagger} \Gamma^{1}+c_{i+\hat{z}}^{\dagger} \Gamma^{3}\right) c_{i}+\text { H.c. }\right)
\end{aligned}
$$

where $c_{i}^{\dagger} \equiv\left(c_{i 1 \uparrow}^{\dagger}, c_{i 2 \uparrow}^{\dagger}, c_{i 1 \downarrow}^{\dagger}, c_{i 2 \downarrow}^{\dagger}\right)$ and $c_{i}$ are the electron creation and annihilation operators at site $i=\left(n_{x}, n_{y}, n_{z}\right)$ for orbits 1 and 2, spin up and spin down. $M$ is the Dirac mass that controls the band inversion, and $B$ is a parameter for controlling gap opening on surfaces. $\Gamma^{0}$ and $\Gamma^{\mu=1-5}$ are, respectively, the four-by-four identity matrix and the five non unique Dirac matrices satisfying $\left\{\Gamma^{\mu}, \Gamma^{\nu}\right\}=2 \delta_{\mu, \nu} \Gamma^{0}$ and $\Gamma^{\mu \nu}=\left[\Gamma^{\mu}, \Gamma^{\nu}\right] /(2 i)$. Here, we choose $\Gamma^{(1-5)}=\left(s_{1} \otimes\right.$

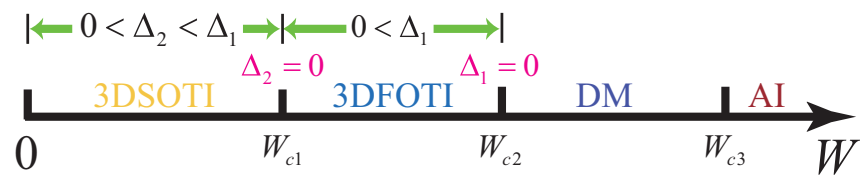

FIG. 1. A generic route of quantum phase transitions in disordered 3DSOTIs. There are four different phases with increasing disorder $W$ : (1) 3DSOTIs characterized by the hinge states localized at the edges; (2) 3DFOTIs identified by the surface states; (3) DMs with wave functions spreading over the whole lattice; (4) AIs where all states are localized. Here, $\Delta_{1}$ and $\Delta_{2}$ are gaps of the bulk and topological surface states, respectively. $W_{c 1}, W_{c 2}$, and $W_{c 3}$ are the three critical disorders separating the four different phases.

$\left.\sigma_{1}, s_{2} \otimes \sigma_{1}, s_{3} \otimes \sigma_{1}, s_{0} \otimes \sigma_{3}, s_{0} \otimes \sigma_{2}\right)$ with the Pauli matrices $s_{\mu}$ and $\sigma_{\mu}$ acting on spin and orbital spaces, respectively. $t=1$ is chosen as the energy unit. $v_{i}$ is a white noise, distributing uniformly in the range of $[-W / 2, W / 2]$.

\section{CLEAN CASE}

In the absence of disorder $(W=0)$, Hamiltonian (1) was well studied [20,37] and can be block diagonalized in the momentum space $H=\sum_{k} c_{k}^{\dagger} h(\boldsymbol{k}) c_{\boldsymbol{k}}$ with

$$
h(\boldsymbol{k})=\sum_{\mu=1}^{4} d_{\mu}(\boldsymbol{k}) \Gamma^{\mu}+B \Gamma^{31} .
$$

Here, $d_{1}(\boldsymbol{k})=t \sin k_{2}, d_{2}(\boldsymbol{k})=M-t \sum_{i=1-3} \cos k_{i}, d_{3}(\boldsymbol{k})=t$ $\sin k_{3}$, and $d_{4}(\boldsymbol{k})=t \sin k_{1}$. For $B=0$ and $1<M<3$, Eq. (2) describes a reflection-symmetric strong 3DFOTI with reflection plane on $x=0$ [38]. The Hamiltonian does not change under the reflection symmetry of $\Gamma^{54}=s_{0} \otimes \sigma_{1}$, i.e., $\Gamma^{54} h\left(k_{1}, k_{2}, k_{3}\right) \Gamma^{54}=h\left(-k_{1}, k_{2}, k_{3}\right)$. According to the bulk-boundary correspondence, the nontrivial bulk topology guarantees the appearance of the gapless surface states on the self-reflected surfaces, e.g., $H_{\text {surface }}=v_{1} k_{1} \sigma_{3}+v_{2} k_{2} \sigma_{1}$ on $z=0$, whereas the gapped surface states on the nonreflected surfaces [39-42]. If two nonreflection surfaces encounter at the reflection plane under a sharp angle, the last term $B \Gamma^{31}$ leads to the band inversion of surface states as well as the emergence of hinge states at their boundary [20,37]. Thus, Hamiltonian (1) supports a 3DSOTI.

To visualize the above descriptions, we plot the energy spectrum $E\left(k_{3}\right)$ of a rectangle bar sample with OBCs on the surfaces perpendicular to (110) and (11) and the PBC along the $z$ direction for $M=2, B=0$ [Fig. 2(a)] and $M=$ $2, B=0.2$ [Fig. 2(b)] where the colors encode the information of the common logarithmic of the participation ratio $p_{2}$. With the help of $\log _{10} p_{2}$, one can easily identify the hinge $\left(\log _{10} p_{2}<1\right)$, surface $\left(\log _{10} p_{2} \sim 1\right)$, and bulk states $\left(\log _{10} p_{2} \sim 2\right.$ ). Clearly, for $B=0$, topological surface states exist in the bulk gap, i.e., $E \in\left[-\Delta_{1} / 2, \Delta_{1} / 2\right]$, whereas for $B=0.2$, surface states are gapped in $E \in\left[-\Delta_{2} / 2, \Delta_{2} / 2\right]$ and the hinge states emerge in the gap, exactly the same as reported results [20,37]. This can also be seen from the distribution of wave function of $E=0$ shown in Figs. 2(c) and $2(\mathrm{~d})$. 

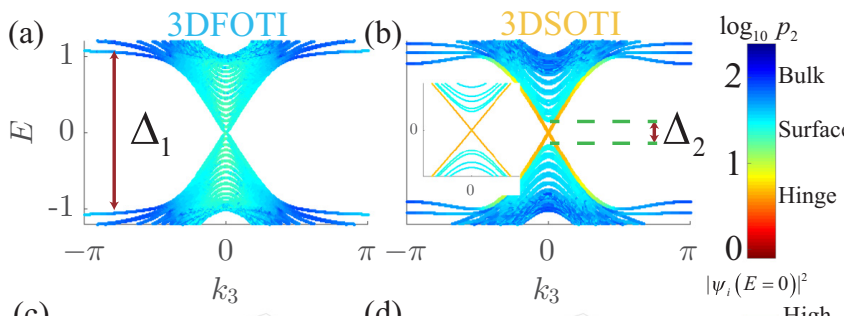

(c)

$k_{3}$

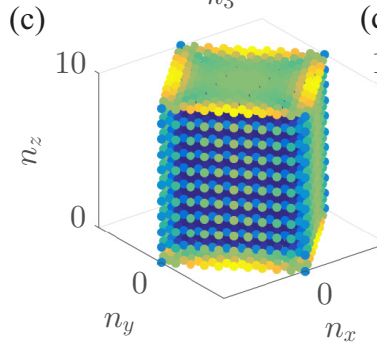

(d)

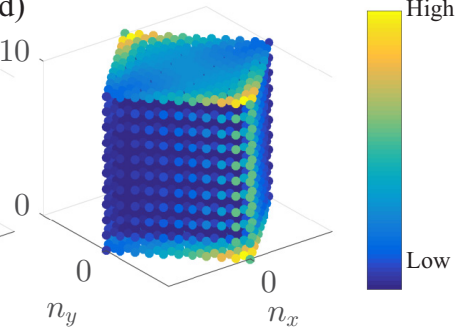

FIG. 2. (a) $E\left(k_{3}\right)$ for $L=32, M=2, B=0$ and (b) $B=0.2$. The periodic boundary condition (PBC) is applied along the $z$ direction and open boundary conditions (OBCs) are used on the (110) and (11̄0) surfaces. Color map $\log _{10} p_{2}$. The orange/red, cyan/green, and blue colors are, respectively, for surface, hinge, and bulk states as denoted by the color bar. The inset in (b) is the enlargement of the zero-energy regime. (c) and (d) Spatial distribution of the wave function (OBCs on all surfaces) of the $E=0$ state, $\left|\psi_{i}\right|^{2}=\sum_{p=1}^{4}\left|\psi_{i, p}\right|^{2}$ for (c) $L=10$ and $B=0$ and (d) $B=0.2$.

\section{DISORDER-INDUCED PHASE TRANSITIONS}

To see how four different phases illustrated in Fig. 1 appear under disorders, we set $M=2, B=0.2$ and vary $W$. First, we demonstrate that 3DSOTIs in clean limit persist to a finite disorder $W_{c 1}$ and, then, become 3DFOTIs for $W>W_{c 1}$ by calculating the dimensionless conductances and density of states. Then, we show that a second quantum phase transition from 3DFOTIs to DMs at a higher-disorder $W_{c 2}$ through the analysis of the change of $E=0$-state distribution on surfaces (explained below) and density of bulk states, which agree well with the SCBA calculations. Finally, we illustrate a third quantum phase transition from DMs to AIs happens at $W_{c 3}$ that is larger than $W_{c 2}$.

\section{A. 3DSOTI-to-3DFOTI}

We consider the Hamiltonian (1) on a bar of size $(L / \sqrt{2} \times$ $L / \sqrt{2} \times L)$. The OBCs on the surfaces perpendicular to (110), (110), and (001) are assumed if not specified otherwise. Note that hinge states in 3DSOTIs are chiral, the conductance through them must be quantized with zero fluctuations [20] if there is no other conduction channels exist at the Fermi level. This feature can be used to distinguish a 3DSOTI hinge state from others. Thus, we compute the two-terminal dimensionless conductances $g_{L}$ of a disordered bar connected to two semi-infinite leads along the $z$ direction. The dimensionless conductance is given by $g_{L}=\operatorname{Tr}\left[T T^{\dagger}\right]$ with $T$ being the transmission matrix [43]. The Fermi energy is fixed at $E=$ 0.02 to focus on the hinge states. Figures 3(a) and 3(b) show, respectively, the sample-averaged dimensionless conductance $\left\langle g_{L}\right\rangle$ and the conductance fluctuation $\delta g_{L}=\left(\left\langle g_{L}^{2}\right\rangle-\left\langle g_{L}\right\rangle^{2}\right)^{1 / 2}$ as a function of disorder $W$ for various sizes from $L=20$

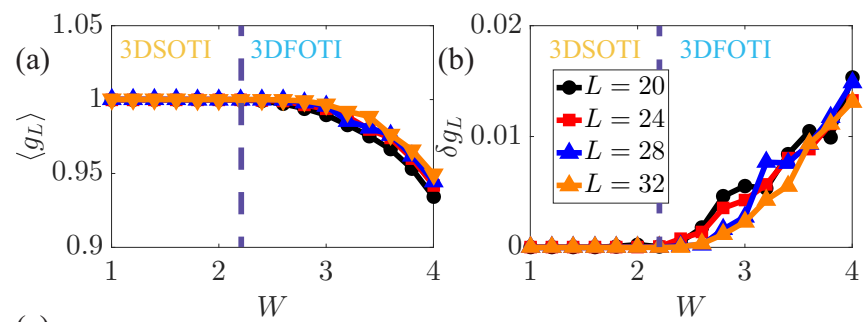

(c)

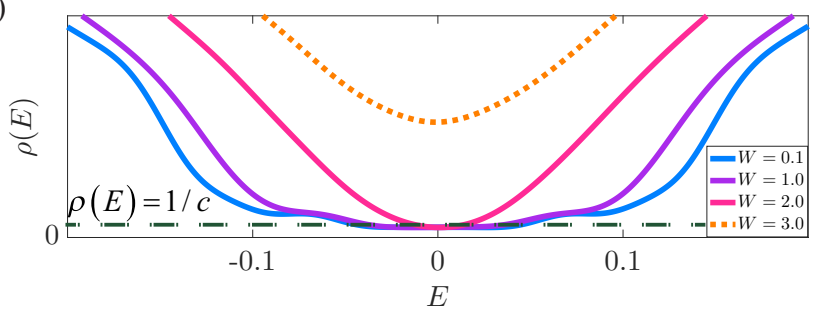

FIG. 3. (a) and (b), (a) $\left\langle g_{L}\right\rangle$ and (b) $\delta g_{L}$ as a function of $W$ for $E=0.02$ and various $L$ 's. The dashed lines denote $W_{c 1}$. (c) $\rho(E)$ for $E \in[-0.15,0.15], L=66$ and various $W$. The solid (dotted) lines are for $W \leqslant W_{c 1}\left(W>W_{c 1}\right)$. The dashed line guides the eyes for a nonzero constant $\rho(E)=1 / c$.

to $L=32$. Clearly, all $\left\langle g_{L}\right\rangle$ 's are exactly quantized at 1 for $W<W_{c 1} \simeq 2.2$ with zero conductance fluctuation, a typical feature of hinge states. Beyond $W_{c 1},\left\langle g_{L}\right\rangle$ is not quantized and $\delta g_{L} \neq 0$. $\left\langle g_{L}\right\rangle$ increases with $L$, an indication of states of $E=0.02$ being extended. As demonstrated later, these states are surface states, and the system is a 3DFOTI.

The dispersion relation of the hinge states in clean 3DSOTIs is linear in $k_{3}$, i.e., $E_{\text {hinge }}= \pm c k_{3}$, see Fig. 2(b). Thus, its contribution to the density of states (DOS) is a constant, i.e., $\rho(E)=1 / c$. Interestingly, the average DOS of disordered 3DSOTI, defined as $\rho(E)=\left\langle 1 /\left(4 L^{3}\right) \sum_{q} \sum_{p=1}^{4} \delta\left(E-E_{p, q}\right)\right\rangle$ and obtained from the kernel polynomial method [44] is a disorder-independent constant. Average $\rho(E)$ of the disordered bar of $L=66$ for various $W$ 's are plotted in Fig. 3(c). Apparently, the width of constant DOS becomes smaller as $W$ increases. This is expected since disorders tend to reduce the gap $\Delta_{2}$. For large enough disorders $W>W_{c 1}$, the constant plateau of $\rho(E)$ disappears when the gap $\Delta_{2}$ of the surface state vanishes and the system becomes a 3DFOTI. To summarize, constant DOS is another fingerprint of the disordered 3DSOTI.

\section{B. 3DFOTI-to-DM}

After establishing the existence of 3DSOTIs for $W<W_{c 1}$, we would like to show, now, that the system is a 3DFOTI for $W \in\left[W_{c 1}, W_{c 2}\right]$ where the zero-energy states are the surface states rather than the hinge states and becomes a DM for $W>$ $W_{c 2}$. Note that the wave function of $E=0$ of both 3DFOTIs and 3DSOTIs are highly localized at system boundaries either on the surfaces in a 3DFOTI or on hinges in a 3DSOTI, in contrast to be extended over the whole systems in a DM. Therefore, we use the following quantity to distinguish states in 3DSOTIs and 3DFOTIs from those in DMs:

$$
\zeta_{W, L}=\sum_{i \in \text { Surface }} \sum_{p=1}^{4}\left|\psi_{i, p}(E=0)\right|^{2},
$$



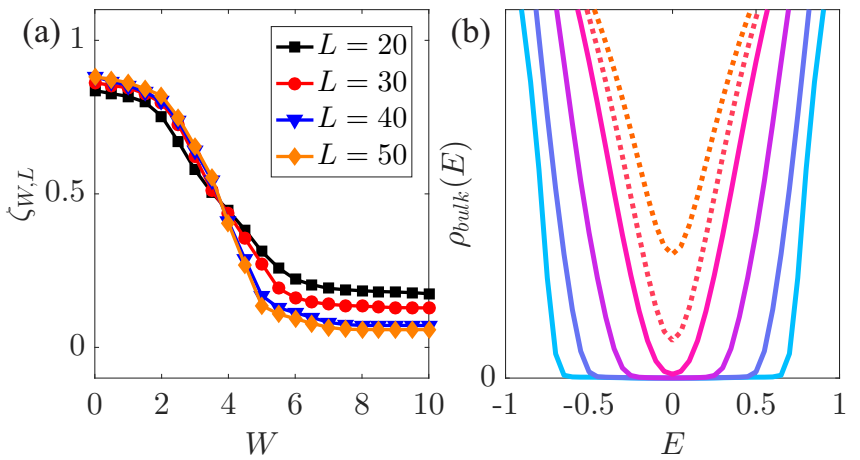

FIG. 4. (a) $\zeta_{W, L}$ vs $W$ for various $L$ 's. (b) $\rho_{\text {bulk }}(E)$ for various $W$ (the solid and the dashed lines are for $W=1-4<W_{c 2}$ and $W=$ $4.5,5>W_{c 2}$, respectively).

with the first summation over all sites on the surfaces. $\zeta_{W, L}$ describes the wave-function distribution of the zero-energy states on surfaces. As expected, $\zeta_{W, L=\infty}$ is a finite nonzero constant for 3DSOTIs and approaches zero for a DM since the ratio of number of surface sites to that of bulk sites goes to zero. For a fixed $W, \zeta_{L, W}$ should increase with $L$ for 3DFOTIs and 3DSOTIs and decreases with $L$ for the DM. They should intersect at critical disorder $W_{c 2}$. Numerically, we use the retarded Lanczos method to find the eigenfunction of the level nearest to $E=0$ of the disordered bar and calculate $\zeta_{W, L}$. In our scenario, we first use the KWANT package [45] to construct a Hamiltonian matrix $H$ out of tight-binding model Eq. (1). We, then, solve the eigenequation $H \psi=E \psi$ using the SCIPY library [46] to obtain the required eigenenergies and eigenfunctions.

To substantiate the above assertion, we evaluate the sample-averaged surface density $\zeta_{W, L}$ for various $L$ 's ranging from 20 to 50 and various $W$ 's. The results are plotted in Fig. 4(a). A phase transition, between the boundary states $\left(d \zeta_{W, L} / d L>0\right)$ and the bulk states $\left(d \zeta_{W, L} / d L<0\right)$ at $W=$ $W_{c 2} \simeq 4$ at which all $\zeta_{W, L}$ curves cross, can be clearly seen. Since $W_{c 2}>W_{c 1}$, the 3DFOTIs, where wave function of $E=$ 0 is localized on the surface rather than the edge of the bar as illustrated in Appendix A, exist between the 3DSOTIs at weak disorders $W<W_{c 1}$ and the DMs beyond $W_{c 2}$ ( $W>$ $W_{c 2}$ ). Figure 4(a) is, thus, a verification of the existence of 3DFOTI-to-DM quantum phase transitions such that $E=0$ states for $W_{c 1}<W<W_{c 2}$ and $W>W_{c 2}$ belong, respectively, to the 3DFOTIs and the DMs in the thermodynamic limit of $L \rightarrow \infty$.

The 3DFOTI-DM transition happens when the bulk gap closes. To substantiate it, we calculate $\rho_{\text {bulk }}(E)$ for the disordered bar of $L=66$ with PBCs along all directions so that all boundary states (surfaces or hinges) are eliminated, and only bulk states can contribute to the DOS. The DOS for various disorders are displayed in Fig. 4(b). As expected, $\rho_{\text {bulk }}(0)=$ 0 below the critical disorder of $W_{c 2} \simeq 4.0$ and $\rho_{\text {bulk }}(0) \neq 0$ beyond $W_{c 2}$, in contrast to nonzero $\rho(E)$ around $E=0$ in Fig. 3(c) for $W<W_{c 2}$. Figure 4(a) demonstrates from a different angle that nonzero $\rho(E)$ around $E=0$ in Fig. 3(c) is from either hinge states of 3DSOTI or surface states of 3DFOTI. The estimate of the critical disorder strength is consistent with that by the finite-size analysis of $\zeta_{W, L}$.
We carry out the SCBA calculations to further understand the disorder effects $[29,30,47,48]$. The self-energy is $\Sigma(E)=$ $\left(W^{2} / 12 N\right) \sum_{k}\left[(E+i 0) \Gamma^{0}-h(\boldsymbol{k})-\Sigma(E)\right]^{-1}$, where $N$ is the total number of sites. For simplicity, the $B \Gamma^{31}$ term $(B \ll$ $M$ in this paper) is neglected, and $\Sigma$ can be expressed as $\Sigma=\sum_{\mu=0}^{4} \Sigma_{\mu} \Gamma^{\mu}$. For $E=0, \Sigma_{0}=-i(1 / \tau)$ is a pure imaginary number, with $\tau$ being the lifetime of the zero-energy bulk states, i.e., $\rho_{\text {bulk }}(E=0) \propto(1 / \tau)$. After some algebra (see Appendix B), we obtain

$$
\frac{1}{\tau}=\frac{1}{\tau} \frac{W^{2}}{12 N} \sum_{k} \frac{1}{\sum_{\mu=1}^{4}\left[d_{\mu}(\boldsymbol{k})+\Sigma_{\mu}(0)\right]^{2}+(1 / \tau)^{2}},
$$

with $\Sigma_{1,3,4}=0$. Here, the summation is taken over the first Brillouin zone (BZ). The solutions of Eq. (4) can be either $1 / \tau=0$ for $W \leqslant W_{c 2}$ or $1 / \tau \neq 0$ for $W>W_{c 2}$. The former corresponds to either 3DFOTIs or 3DSOTIs where $\Delta_{1} \neq 0$ and $\rho_{\text {bulk }}(0)=0$, whereas the later is for the DMs with $\Delta_{1}=$ 0 and $\rho_{\text {bulk }}(0) \neq 0$. The critical disorder $W_{c 2}$ is given by the gap equation [48],

$$
1=\frac{W_{c 2}^{2}}{12 N} \sum_{\boldsymbol{k}} \frac{1}{\sum_{\mu=1}^{4}\left[d_{\mu}(\boldsymbol{k})+\Sigma_{\mu}(0)\right]^{2}} .
$$

Numerically, we obtain $W_{c 2} \simeq 3.7$ for $M=2$ and $B=0.2$, consistent with the estimates from $\zeta_{W, L}$ and $\rho_{\text {bulk }}(E)$. According to the SCBA, $M$ is renormalized by the disorder as $\tilde{M}=M+\Delta$ with

$$
\Delta=-\frac{W^{2}}{12 N} \sum_{\boldsymbol{k}} \frac{d_{2}(\boldsymbol{k})+\Delta}{\sum_{\mu=1}^{4}\left[d_{\mu}(\boldsymbol{k})+\Sigma_{\mu}(0)\right]^{2}},
$$

such that the phase boundary among 3DSOTIs, 3DFOTIs, and the topological trivial phase are shifted by disorders.

\section{DM-to-AI}

The extended states in the DMs are eventually localized by strong disorders. To investigate the nature of this Anderson localization transition and its associated universality class, we compute the PR $p_{2}(E=0, W)$, which measures how many lattice sites are occupied by the wave function of $E=0$ [49-51]. Near the critical disorder $W_{c 3}$ of the Anderson localization transitions, $p_{2}$ satisfies the one-parameter scaling function $[52,53]$,

$$
p_{2}(W)=L^{D}\left[f(L / \xi)+C L^{-y}\right],
$$

where $f(x)$ is the unknown scaling function, $C$ and $y>0$ are a constant and the exponent of the irrelevant variable, respectively. The correlation length $\xi$ diverges at $W_{c 3}$ as $\xi \propto \mid W-$ $\left.W_{c 3}\right|^{-v}$ with critical exponent $v . D$ is the fractal dimension of critical wave functions which occupy a subspace of dimensionality smaller than the embedded space dimension $d=3$. By defining $Y_{L}(W)=p_{2} L^{-D}-C L^{-y}$, we use the following criteria to identify an Anderson localization transition [53]: (1) $d Y_{L}(W) / d L>0\left[d Y_{L}(W) / d L<0\right]$ for the DMs (AIs). (2) Near $W_{c 3}, Y_{L}(W)$ of different $L$ 's collapse into a single curve of $f(x)$. 


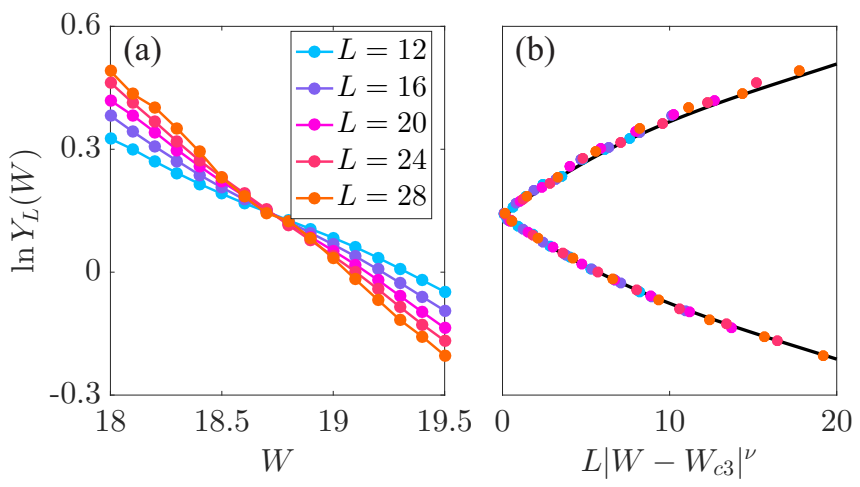

FIG. 5. (a) $\ln Y_{L}(W)$ for $E=0$ and various $L$ 's. (b) Scaling function $\ln Y_{L}(W)=\ln f\left(x=L\left|W-W_{c 3}\right|^{\nu}\right)$ for the Anderson localization transition.

Near $W_{c 3}$, the calculated $\ln Y_{L}(W)$ and $\ln f(x)$ are displayed in Figs. 5(a) and 5(b), respectively. Data in Fig. 5(a) give $W_{c 3}=18.73 \pm 0.03$, and $d Y_{L}(W) / d L$ is always positive (negative) for $W<W_{c 3}\left(W>W_{c 3}\right)$, indicating the system is a DM (AI). Following the well-established procedure [53], we find $D=1.7 \pm 0.2$, and $v=1.5 \pm 0.2$ (see Appendix C for more details). The obtained $v$ and $D$, characterizing the universality class of transitions, are consistent with previous estimations for the 3D Gaussian unitary ensemble [54].

\section{PHASE DIAGRAMS}

We would like to depict a more inclusive general phase diagram of the model by varying both $M$ and $W$ for a fixed $B=0.2$ in this section, in contrast of varying $W$ only for fixed $M$ and $B$. Let us discuss the clean case first. For $M>0$, there are four critical points at $M=1 \pm B$ and $3 \pm B$ that separate three different phases from each other (see the red dots in Fig. 7). For $M \in[1-B, 1+B](M \in[3-B, 3+B])$, Eq. (2) is a gapless Weyl semimetal (WSM) with three (one) pairs of Weyl nodes in the first BZ, whereas the band gap is nonzero for $0<M<1-B, 1+B<M<3-B$, and $M>$ $3+B$ (see Appendix D for more details). As $M$ increases, we find that the system is a 3DSOTI for $0<M<1-B$,

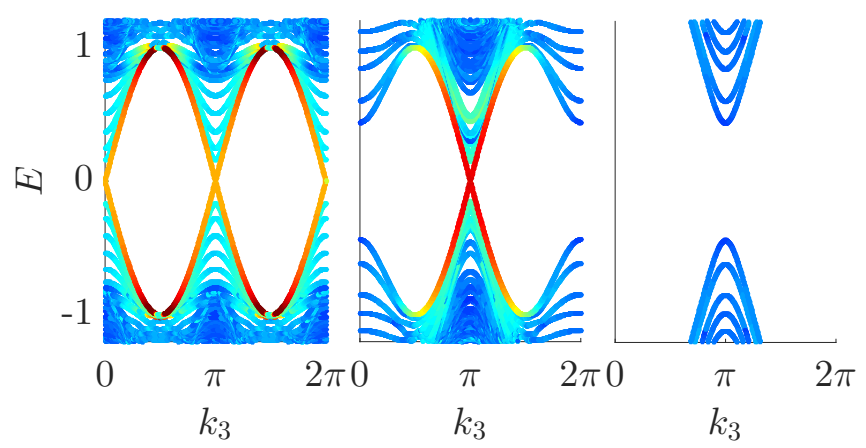

FIG. 6. Energy spectrum $E\left(k_{3}\right)$ for $L=32, B=0.2$ and three typical $M$ 's. Left: $M=0$ (3DSOTI); middle: $M=1.5$ (3DSOTI); right: $M=3.5$ (normal insulator). Colors encode $\log _{10} p_{2}$, and the color bar is the same as those in Figs. 2(a) and 2(b).

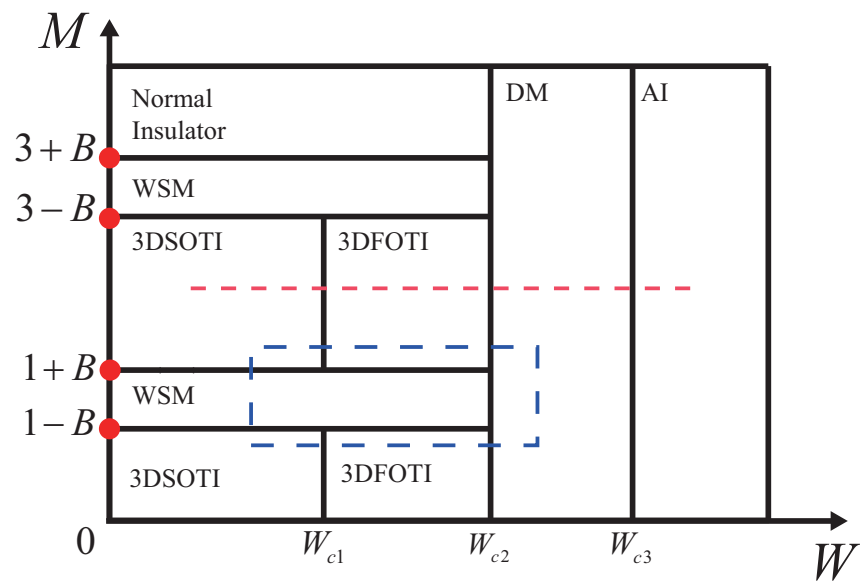

FIG. 7. Schematic of the general phase diagrams on the $W-M$ plane. $M$ is the Dirac mass that drives quantum phase transitions among 3DFOTIs, 3DSOTIs, WSMs, and normal insulators. $W$ measures the disorder strength. $B$ is the tuning parameter for the term that causes the band inversions in surface states of 3DFOTIs. We only consider $B=0.2<1$ here. The phase boundaries are the sketches only.

enters a WSM at $M=1-B$, reenters into the 3DSOTI at $M=1+B$, becomes a WSM again for $3-B<M<3+B$, and is a normal gapped insulator for $M>3+B$ where there is no band inversion for surface states. Remarkably, there are two pairs of one-dimensional helical edge channels (hinge states) for $M<1-B$, indicating the occurrence of band inversions for two Dirac cones. Consequently, the two-terminal conductance should be quantized at $2 e^{2} / h$ for $M<1-B$. On the other hand, there is only one surface Dirac cone for $1+B<M<3-B$, the conductance of the 3DSOTI is, thus, quantized to $e^{2} / h$. The one or two Dirac cones show clearly in the spectrum $E\left(k_{3}\right)$ of a Hall bar with OBCs on the surfaces perpendicular to (10) and (11 10$)$ and PBCs along the $z$ direction. Figures 6(a)-6(c) plot $E\left(k_{3}\right)$, respectively, for $M=0,1.5,3.5$.

Naturally, we expect that the 3DSOTIs, due to the band inversion of surface states with both one or two Dirac cones shown in Figs. 6(a) and 6(b), are robust against weak disorders. With the increase in $W$, a transition from a 3DSOTI to a 3DFOTI occurs at a critical disorder $W_{c 1}$ where the gap of surface states $\Delta_{2}$ closes but the gap of bulk states $\Delta_{1}$ remains open. At some higher critical disorders $W_{c 2}, \Delta_{1}=0$ such that a transition from 3DFOTIs to DMs occurs. Finally, an Anderson localization transition from DMs to AIs occurs at large enough disorders $W_{c 3}$. All these features can be seen from a schematic phase diagram in Fig. 7. In addition to the four phases shown in Fig. 1, there are two more phases: the WSMs characterized by paired Weyl nodes in the clean limit and the normal gapped insulators. Noticeably, the phase boundary of the disordered WSMs is still an issue under debate, e.g., whether the WSMs can exist in finite disorders [52] and whether there is a direct WSM-to-DM transition without the intermediated Chern insulator phase $[47,48,53]$ or two quantum phase transitions of WSM-to-CI-to-DM with 


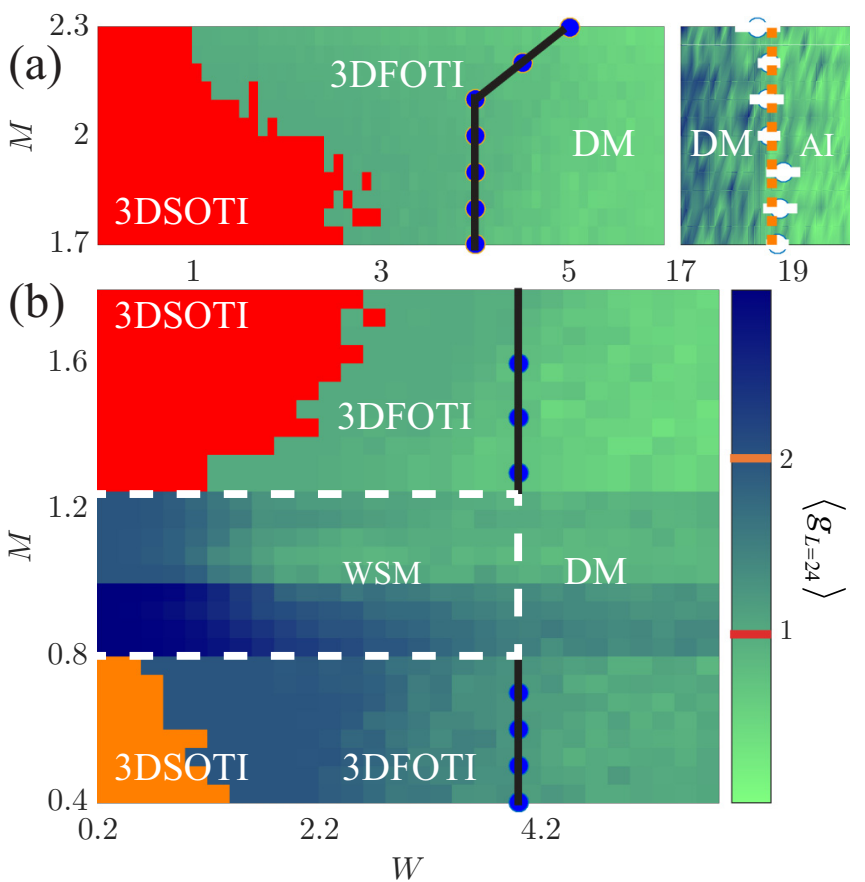

FIG. 8. Phase diagram on the $W-M$ plane displaying the occurrences of 3DSOTIs, 3DFOTIs, DMs, and AIs for different parameter regimes of Fig. 7: (a) along the red line and (b) within the blue rectangle. Colors encode $\left\langle g_{L=24}(W, M)\right\rangle$. The 3DSOTIs are characterized by the quantized conductances. The boundaries between 3DFOTIs and DMs (black solid lines) are determined by finite-size scaling analysis of $\zeta_{W, L}$. The boundary between DMs and AIs (orange dashed line) is given by scaling analysis of $p_{2}(W, L)$. White dashed lines show the boundary of disordered WSMs schematically, which is still controversial.

increasing disorders [55]. However, this challenging problem is not the focus of this paper.

We have partly confirmed the general phase diagram through exhaustive numerical calculations of different $M$ 's and $W$ 's and a fixed $B=0.2$. We first consider the phase transitions along the red line in Fig. 7. A phase diagram on the $W-M$ plane displaying the existences of the 3DSOTI, 3DFOTI, DM, and AI phases is shown in Fig. 8(a). $W_{c 1}$ and $W_{c 2}$, respectively, and monotonically decrease and increase with $M$ whereas $W_{c 3}$ does not depend on $M$. Although the phase boundaries, i.e., $W_{c 1}$. $W_{c 2}$, and $W_{c 3}$, depend on the details of a model, the physics in Fig. 8(a) and Fig. 1 is general.

We provide additional supports to the general phase diagram by probing the blue dashed rectangle regime in Fig. 7. The results are shown in Fig. 8(b). As expect, the dimensionless conductance $\left\langle g_{L=24}\right\rangle$ are exactly quantized at 2 and 1 for the 3DSOTIs with $0<M<1-B$ and $1+B<M<3-B$, respectively. Indeed, it is so as shown by the orange and red colors in Fig. 8(b). Although transitions from 3DSOTIs to 3DFOTIs occur at critical disorders $W_{c 1}$ above which the conductances lose the quantization. Further increasing $W$ to $W_{c 2}$, the system undergoes a transition from 3DFOTIs to DMs. The critical disorder $W_{c 2}$ is also determined by the scaling analysis of $\zeta_{W, L}$ (probability for the electron on surfaces) as we did in Fig. 3(a).

\section{CONCLUSION}

In conclusion, a generic route of disorder-induced phase transitions for a crystal 3DSOTI is revealed. As random potential strength increase, the 3DSOTI transforms to a 3DFOTI at a lower weak critical disorder of $W_{c 1}$, followed by a second transition at an intermediate higher critical disorder of $W_{c 2}$ to a DM. The system eventually becomes an AI after a metalto-insulator transition at an even stronger critical disorder of $W_{c 3}$. The 3DSOTI is featured by quantized conductance at $e^{2} / h$ and zero conductance fluctuation as well as the constant density of states, whereas 3DFOTIs are identified by their dominate occupation on surfaces, negligible occupation in the bulk and on hinges. The DM and AI are confirmed by the scaling analysis of participation ratios, and the corresponding Anderson localization transition belongs to the conventional 3D Gaussian unitary class. We believe that such a general route should be held for two-dimensional second-order topological insulators too, but whether there is an intermediate DM phase depends on system symmetries [51].

\section{ACKNOWLEDGMENTS}

This work is supported by the National Natural Science Foundation of China (Grants No. 11774296, No. 11704061, and No. 11974296) and Hong Kong RGC (Grants No. 16301518 and No. 16301619). C.W. acknowledges the kindly help from J. Lu and X. Hu.

\section{APPENDIX A: ADDITIONAL EVIDENCE FOR TRANSITIONS FROM 3DFOTI TO 3DSOTI}

In this Appendix, we show that the occupation probabilities of an electron in the $E=0$ state lie on the hinges (surfaces) if $0<W<W_{c 1}\left(W_{c 1}<W<W_{c 2}\right)$. We define edge occupation probability $\lambda_{W, L}$,

$$
\lambda_{W, L}=\sum_{i \in \text { edge }} \sum_{p=1}^{4}\left|\psi_{i, p}(E=0)\right|^{2},
$$

where $\sum_{i \in \text { edge }}$ is over the two edges of the Hall bars lying on the reflection plane $x=0$. The edge occupation probability $\lambda_{W, L}$ is the order of 1 for a 3DSOTI and negligibly small for a 3DFOTI in the limit of $L \rightarrow \infty$. The calculated $\lambda_{W, L}$ for $W=1,1.5, \ldots, 4$ and $L=2-50$ are plotted in Fig. 9. Indeed, $\lambda_{W, L}$ approaches a finite nonzero number for $W<W_{c 1}$ and decrease with both $W$ and $L$ for $W>W_{c 1} \simeq 2.2$, an obvious feature of transition from a 3DSOTI to a 3DFOTI. It is more convincing if one recalls the featureless behavior of surface occupation probability $\zeta_{W, L}$ around $W_{c 1} \simeq 2.2$ shown in Fig. 4(a). This extra property of hinge occupation probability is another support of 3DSOTI-to-3DFOTI transition at $W_{c 1}$.

\section{APPENDIX B: SELF-CONSISTENT BORN APPROXIMATION}

Within the framework of the self-consistent Born approximation [29], the self-energy $\Sigma$ reads

$$
\Sigma(E)=\frac{W^{2}}{12 N} \sum_{\boldsymbol{q}} \bar{G}(\boldsymbol{q}, E),
$$




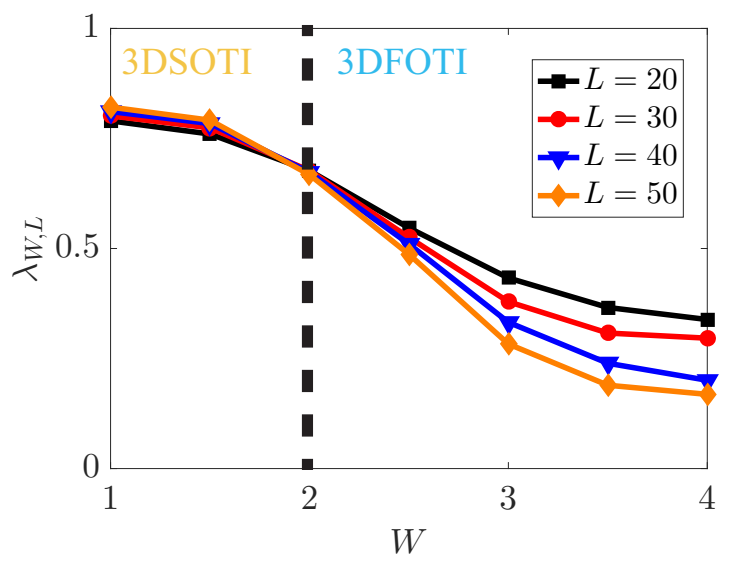

FIG. 9. $\lambda_{W, L}$ for $M=2, B=0.2$, and various $W$ 's and $L$ 's. The dashed line locates the $W_{c 1}$.

with the summation $\sum_{q}$ taking over the first BZ. Now, our task is to solve

$$
\bar{G}^{-1}(\boldsymbol{k}, E)=G_{0}^{-1}(\boldsymbol{k}, E)-\frac{W^{2}}{12 N} \sum_{\boldsymbol{q}} \bar{G}(\boldsymbol{q}, E),
$$

with the free Green's function $G_{0}(\boldsymbol{k}, E)=\left[(E+i 0) \Gamma_{0}-\right.$ $h(\boldsymbol{k})]^{-1}$. In order to avoid a complicated calculation, we omit the $B \Gamma^{31}$ term (since $B \ll 1$ ) such that the free Green's function is the combination of $\Gamma^{0}$ and $\Gamma^{\nu}$, i.e., $G_{0}^{-1}(\boldsymbol{k}, E)=$ $(E+i 0) \Gamma^{0}-\sum_{\mu=1}^{4} d_{\mu}(\boldsymbol{k}) \Gamma^{\mu}$. Since the free Green's function includes the identity matrix and the $\Gamma$ matrices only, we can write the self-energy $\Sigma(E)$ in terms of the identity and $\Gamma$ matrices as well, namely, $\Sigma(E)=\Sigma_{0} \Gamma^{0}+\sum_{\mu=1}^{4} \Sigma_{\mu} \Gamma^{\mu}$. Therefore,

$$
\begin{aligned}
\Sigma(E)= & \frac{W^{2}}{12 N} \sum_{\boldsymbol{q}} \\
& \times \frac{1}{\left(E+i 0-\Sigma_{0}\right) \Gamma_{0}-\sum_{\mu=1}^{4}\left[d_{\mu}(\boldsymbol{q})+\Sigma_{\mu}(E)\right] \Gamma^{\nu}} .
\end{aligned}
$$

By comparing the coefficients of the identity and $\Gamma$ matrices, we find that

$$
\Sigma_{0}(E)=\frac{W^{2}}{12 N} \sum_{\boldsymbol{q}} \frac{\Sigma_{0}-E}{\sum_{\mu=1}^{4}\left[d_{\mu}(\boldsymbol{q})+\Sigma_{\mu}(E)\right]^{2}-\left(E-\Sigma_{0}\right)^{2}}
$$

and

$$
\Sigma_{\mu}(E)=\frac{W^{2}}{12 N} \sum_{q} \frac{-\left[d_{\mu}(\boldsymbol{q})+\Sigma_{\mu}(E)\right]}{\sum_{\mu=1}^{4}\left[d_{\mu}(\boldsymbol{q})+\Sigma_{\mu}(E)\right]^{2}-\left(E-\Sigma_{0}\right)^{2}} .
$$

For $E=0, \Sigma_{0}$ should be a pure imaginary number, i.e., $\Sigma_{0}=$ $i(1 / \tau)$, from Eq. (B4). Thus, we obtain

$$
\frac{1}{\tau}=\frac{1}{\tau} \frac{W^{2}}{12 N} \sum_{\boldsymbol{q}} \frac{1}{\sum_{\mu=1}^{4}\left[d_{\mu}(\boldsymbol{q})+\Sigma_{\mu}(0)\right]^{2}+(1 / \tau)^{2}} .
$$

From Eq. (B6), we can determine the critical disorder $W_{c 2}$ for the transition from 3DFOTIs $(1 / \tau=0)$ to the DMs $(1 / \tau \neq 0)$,

$$
1=\frac{W_{c 2}^{2}}{12 N} \sum_{q} \frac{1}{\sum_{\mu=1}^{4}\left[d_{\mu}(\boldsymbol{q})+\Sigma_{\mu}(0)\right]^{2}} .
$$

Also, the parameter $M$ is renormalized as $\tilde{M}=M+\Delta$ with

$$
\Delta=-\frac{W^{2}}{12 N} \sum_{\boldsymbol{q}} \frac{d_{2}(\boldsymbol{q})+\Delta}{\sum_{\mu=1}^{4}\left[d_{\mu}(\boldsymbol{q})+\Sigma_{\mu}(0)\right]^{2}}
$$

for both 3DSOTIs and 3DFOTIs. On the other hand, since $d_{1,3,4}(\boldsymbol{q}) /\left[D(\boldsymbol{q})^{2}+(1 / \tau)^{2}\right]$ are odd functions of $\boldsymbol{q}$, the integrals of such functions over the first BZ are zeros. Thus, $\Sigma_{1,3,4}=0$.

We use the following scenario to determine $W_{c 2}$ numerically. First, we obtain a numerical solution of Eq. (B8) by the iterative algorithm:

(1) For a given $M$, choose a fixed disorder $W$.

(2) At the first run, set $\Delta_{1}$ by a random seed value. For the following, we use $\Delta_{i-1}$ of the previous run as the seed.

(3) Calculate the right-hand side of Eq. (B8) by $\Delta_{i-1}$ and set it to be $\Delta_{i}$.

(4) Repeat Steps 2 and 3 for some iterations (in general, 50) until $\left|\Delta_{i}-\Delta_{i-1}\right| /\left|\Delta_{i}\right|<\delta_{\text {tol }}$ with $\delta_{\text {tol }}$ being the tolerance.

After determining $\Delta$ by the above algorithm, we, then, calculate the critical disorder $W_{c 2}$ for the quantum phase transition from a 3DFOTI to a DM by Eq. (B7).

\section{APPENDIX C: FINITE-SIZE SCALING ANALYSIS}

The PRs of different sizes follow the one-parameter scaling function,

$$
p_{2}(L, W)=L^{D}\left[f(L / \xi)+C L^{-y}\right],
$$

with $D$ being the fractal dimension, $\xi=\xi(W)$ being the correlation length, $y$ being the exponents of the irrelevant scaling variable, and $C$ being a constant, providing that $W$ is closed to the critical disorder $W_{c 3}$ from DMs to AIs. $f(x)$ is an unknown scaling function, and $\xi$ diverges as a power law near $W_{c 3}$, i.e., $\xi \propto\left|W-W_{c 3}\right|^{-\nu}$ with $v$ being the critical exponent.

To obtain the unknown scaling function $f(x)$, we have expanded it to the fourth order of $x=L\left|W-W_{c 3}\right|^{\nu}$,

$$
\begin{aligned}
f(x)= & F_{0}+F_{1}\left(L\left|W-W_{c 3}\right|^{\nu}\right)+F_{2}\left(L\left|W-W_{c 3}\right|^{\nu}\right)^{2} \\
& +F_{3}\left(L\left|W-W_{c 3}\right|^{\nu}\right)^{3}+F_{4}\left(L\left|W-W_{c 3}\right|^{\nu}\right)^{4},
\end{aligned}
$$

and fitted the numerical data shown in Fig. 4(b) by minimizing the $\chi^{2}$,

$$
\begin{aligned}
\chi^{2}= & \sum_{i=1}^{N_{w}} \sum_{j=1}^{N_{L}} \\
& \times\left(\frac{p_{2}\left(W_{i}, L_{j}\right)-L_{j}^{D}\left[f\left(L_{j}\left|W_{i}-W_{c 3}\right|^{\nu}\right)+C L_{j}^{-y}\right]}{\sigma_{i j}}\right)^{2},
\end{aligned}
$$

where $N_{w}$ and $N_{L}$ are the numbers of disorder strengths and lengths, respectively. $\sigma_{i j}$ is the standard deviation of $p_{2}\left(W_{i}, L_{j}\right)$. The fitting parameters are $W_{c 3}, D, v, C, y, F_{0-4}$. The fitting yields $W_{c 3}=18.73 \pm 0.03, D=1.7 \pm 0.2$, 
$v=1.45 \pm 0.05, C=0.2 \pm 0.1, y=0.7 \pm 0.1, \quad$ and the scaling function $f(x)$ defined by Eq. (C2). The goodness of fit is $Q=0.2>10^{-3}$, indicating the fit is acceptable [56].

\section{APPENDIX D: CLEAN WSM PHASE}

In this Appendix, we substantiate that Eq. (2) is a WSM if $M \in[1-B, 1+B]$ and $M \in[3-B, 3+B]$ by showing that the conduction and the valence bands cross linearly at Weyl nodes in the first BZ. The energy spectrum of Eq. (2) reads

$$
E_{p q}(\boldsymbol{k})=p \sqrt{\left[B+q \sqrt{d_{2}^{2}(\boldsymbol{k})+d_{4}^{2}(\boldsymbol{k})}\right]^{2}+d_{1}^{2}(\boldsymbol{k})+d_{3}^{2}(\boldsymbol{k})} .
$$

Here, $p, q= \pm$ stands for different subbands. To close the gap, one has

$$
\begin{aligned}
B-\sqrt{\left(M-\sum_{i=1-3} \cos k_{i}\right)^{2}+\sin ^{2} k_{1}} & =0, \quad \sin k_{2}=0, \\
\sin k_{3} & =0 .
\end{aligned}
$$

Note that $\sin k_{2}=\sin k_{3}=0$ give $k_{2}=0, \pm \pi$ and $k_{3}=$ $0, \pm \pi$. We would like to find the possible solutions of Eq. (D2) by considering different situations.

(1) $\left(k_{2}, k_{3}\right)=(0,0)$.

In these cases, possible $k_{1}$ and $M$ satisfy $B^{2}=(M-2-$ $\left.\cos k_{1}\right)^{2}+\sin ^{2} k_{1}$, i.e.,

$$
-1 \leqslant \cos k_{1}=\frac{(M-2)^{2}+1-B^{2}}{2(M-2)} \leqslant 1 .
$$

If $(M-2)>0$, one has

$$
\frac{(M-2)^{2}+1-B^{2}}{2(M-2)} \leqslant 1 \rightarrow 3-B \leqslant M \leqslant 3+B .
$$

Therefore, the gap will close at

$$
\boldsymbol{k}_{0}=\left[ \pm \arccos \left(\frac{(M-2)^{2}+1-B^{2}}{2(M-2)}\right), 0,0\right]
$$

for $3-B \leqslant M \leqslant 3+B$. If $M=2$, there is no possible $k_{1}$ and $M$ since $B=0.2 \neq 1$. If $(M-2)<0$, one has

$$
-1 \leqslant \frac{(M-2)^{2}+1-B^{2}}{2(M-2)} \rightarrow 1-B \leqslant M \leqslant 1+B .
$$

Thus, the gap will close at

$$
\boldsymbol{k}_{0}=\left[ \pm \arccos \left(\frac{(M-2)^{2}+1-B^{2}}{2(M-2)}\right), 0,0\right]
$$

for $1-B \leqslant M \leqslant 1+B$.

(2) $\left(k_{2}, k_{3}\right)=(\pi, 0) \quad$ [equivalent to $(-\pi, 0),(0, \pi)$, $(0,-\pi)]$.

Now, we need to find possible $k_{1}$ and $M$ satisfying $B^{2}=$ $\left(M-\cos k_{1}\right)^{2}+\sin ^{2} k_{1}$, i.e.,

$$
-1 \leqslant \cos k_{1}=\frac{M^{2}+1-B^{2}}{2 M} \leqslant 1 .
$$

For $M>0$,

$$
\frac{M^{2}+1-B^{2}}{2 M} \leqslant 1 \rightarrow 1-B \leqslant M \leqslant 1+B .
$$

Therefore, the gap will close if $1-B \leqslant M \leqslant 1+B$ at

$$
\begin{aligned}
\boldsymbol{k}_{0}= & {\left[ \pm \arccos \left(\frac{M^{2}+1-B^{2}}{2 M}\right), \pm \pi, 0\right], } \\
& \times\left[ \pm \arccos \left(\frac{M^{2}+1-B^{2}}{2 M}\right), 0, \pm \pi\right] .
\end{aligned}
$$

For $M=0$, no $k_{1}$ and $M$ exist since $B \neq 1$. For $M<0$,

$$
-1 \leqslant \frac{M^{2}+1-B^{2}}{2 M} \rightarrow-1-B \leqslant M \leqslant-1+B .
$$

In these cases, the gap will close if $-1-B \leqslant M \leqslant-1+B$ at

$$
\begin{aligned}
\boldsymbol{k}_{0}= & {\left[ \pm \arccos \left(\frac{M^{2}+1-B^{2}}{2 M}\right), \pm \pi, 0\right], } \\
& \times\left[ \pm \arccos \left(\frac{M^{2}+1-B^{2}}{2 M}\right), 0, \pm \pi\right] .
\end{aligned}
$$

(3) $\left(k_{2}, k_{3}\right)=(\pi, \pi)$ the same as $(\pi,-\pi),(-\pi, \pi)$, $(-\pi,-\pi)]$.

Now, it is required that $B^{2}=\left(M+2-\cos k_{1}\right)^{2}+\sin ^{2} k_{1}$, i.e.,

$$
-1 \leqslant \cos k_{1}=\frac{(M+2)^{2}+1-B^{2}}{2(M+2)} \leqslant 1 .
$$

If $M+2>0$,

$$
\frac{(M+2)^{2}+1-B^{2}}{2(M+2)} \leqslant 1 \rightarrow-1-B \leqslant M \leqslant-1+B .
$$

Therefore, the gap closes for $-1-B \leqslant M \leqslant-1+B$ at

$$
\boldsymbol{k}_{0}=\left[ \pm \arccos \left(\frac{(M+2)^{2}+1-B^{2}}{2(M+2)}\right), \pm \pi, \pm \pi\right] .
$$

For $M+2=0$, the gap is open since $B=0.2 \neq 1$. For $M+$ $2<0$,

$$
-1 \leqslant \frac{(M+2)^{2}+1-B^{2}}{2(M+2)} \rightarrow-3-B \leqslant M \leqslant-3+B .
$$

Therefore, the gap closes for $-3-B \leqslant M \leqslant-3+B$ at

$$
\boldsymbol{k}_{0}=\left[ \pm \arccos \left(\frac{(M+2)^{2}+1-B^{2}}{2(M+2)}\right), \pm \pi, \pm \pi\right] \text {. }
$$

We summarize all those results in Table I.

We, then, discuss the dispersion near the band touching points $\boldsymbol{k}_{0}$ by expanding the dispersion Eq. (D1) about $\boldsymbol{k}=\boldsymbol{k}_{0}+\delta \boldsymbol{k}$. Let us consider the parameter regime $M \in$ $[3-B, 3+B]$ where the bulk touching points are (refer Table I)

$$
\begin{aligned}
\boldsymbol{k}_{0} & =\left[ \pm \arccos \left(\frac{(M-2)^{2}+1-B^{2}}{2(M-2)}\right), 0,0\right] \\
& =\left( \pm \arccos k_{0,1}, 0,0\right) .
\end{aligned}
$$


TABLE I. This table shows the regimes that Eq. (2) is gapless as well as the band touching points. Here, we fix $B=0.2$.

\begin{tabular}{lcc}
\hline \hline Parameter regimes & Band-closing points $\boldsymbol{k}_{0}$ & Number of band-closing points in the first BZ \\
\hline$M \in[3-B, 3+B]$ & {$\left[ \pm \arccos \left(\frac{(M-2)^{2}+1-B^{2}}{2(M-2)}\right), 0,0\right]$} & 2 \\
$M \in[1-B, 1+B]$ & {$\left[ \pm \arccos \left(\frac{M^{2}+1-B^{2}}{2 M}\right), \pm \pi, 0\right],\left[ \pm \arccos \left(\frac{M^{2}+1-B^{2}}{2 M}\right), 0, \pm \pi\right]$} & 6 \\
$M \in[-1-B,-1+B]$ & {$\left[ \pm \arccos \left(\frac{(M-2)^{2}+1-B^{2}}{2(M-2)}\right), 0,0\right]$} & 6 \\
$M \in[-3-B,-3+B]$ & {$\left[ \pm \arccos \left(\frac{M^{2}+1-B^{2}}{2 M}\right), \pm \pi, 0\right],\left[ \pm \arccos \left(\frac{M^{2}+1-B^{2}}{2 M}\right), 0, \pm \pi\right]$} & 2 \\
\hline \hline
\end{tabular}

The conduction and valence bands read

$E_{p-}(\delta \boldsymbol{k})=p \sqrt{\left(\frac{(M-2) \sin k_{0,1}}{B}\right)^{2}\left(\delta k_{1}\right)^{2}+\left(\delta k_{2}\right)^{2}+\left(\delta k_{3}\right)^{2}}$,

(D10) which cross linearly at the band touch points. Likewise, we obtain the same dispersion as Eq. (D10) for $\delta \boldsymbol{k}=$ $\boldsymbol{k}-\left(-k_{0,1}, 0,0\right)$. Therefore, for $B \neq 0$ and $M \in[3-B, 3+$ $B]$, the system is a WSM with linear dispersions near $\left( \pm k_{0,1}, 0,0\right)$. Following the same approach, we find the system to be a WSM for $-3-B \leqslant M \leqslant-3+B$ (with a pair of Weyl nodes) as well as $-1-B \leqslant M \leqslant-1+B$ and $1-B \leqslant$ $M \leqslant 1+B$ (with three pairs of Weyl nodes).
[1] C. L. Kane and E. J. Mele, $Z_{2}$ Topological Order and the Quantum Spin Hall Effect, Phys. Rev. Lett. 95, 146802 (2005).

[2] B. A. Bernevig, T. L. Hughes, and S.-C. Zhang, Quantum spin hall effect and topological phase transition in $\mathrm{HgTe}$ quantum wells, Science 314, 1757 (2006).

[3] M. König, S. Wiedmann, C. Bröune, A. Roth, H. Buhmann, L. W. Molenkamp, X.-L. Qi, and S.-C. Zhang, Quantum spin hall insulator state in HgTe quantum wells, Science 318, 766 (2007).

[4] M. Z. Hasan and C. L. Kane, Colloquium: Topological insulators, Rev. Mod. Phys. 82, 3045 (2010).

[5] X.-L. Qi and S.-C. Zhang, Topological insulators and superconductors, Rev. Mod. Phys. 83, 1057 (2011).

[6] X. Wan, A. M. Turner, A. Vishwanath, and S. Y. Savrasov, Topological semimetal and Fermi-arc surface states in the electronic structure of pyrochlore iridates, Phys. Rev. B 83, 205101 (2011).

[7] R. Süsstrunk and S. D. Huber, Observation of phononic helical edge states in a mechanical topological insulator, Science 349, 47 (2015).

[8] F. Li, X. Huang, J. Lu, J. Ma, and Z. Liu, Weyl points and Fermi arcs in a chiral phononic crystal, Nat. Phys. 14, 30 (2018).

[9] M. Serra-Garcia, V. Peri, R. Susstrunk, O. R. Bilal, T. Larsen, L. G. Villanueva, and S. D. Huber, Observation of a phononic quadrupole topological insulator, Nature (London) 555, 342 (2018).

[10] X. Ni, M. Weiner, A. Alú, and A. B. Khanikaev, Observation of higher-order topological acoustic states protected by generalized chiral symmetry, Nat. Mater. 18, 113 (2019).

[11] H. Fan, B. Xia, L. Tong, S. Zheng, and D. Yu, Elastic Higher-Order Topological Insulator with Topologically Protected Corner States, Phys. Rev. Lett. 122, 204301 (2019).
[12] A. B. Khanikaev, S. H. Mousavi, W.-K. Tse, M. Kargarian, A. H. MacDonald, and G. Shvets, Photonic topological insulators, Nat. Mater. 12, 233 (2013).

[13] L. Lu, Z. Wang, D. Ye, L. Ran, L. Fu, J. D. Joannopoulos, and M. Soljačić, Experimental observation of Weyl points, Science 349, 622 (2015).

[14] A. El Hassan, F. K. Kunst, A. Moritz, G. Andler, E. J. Bergholtz, and M. Bourennane, Corner states of light in photonic waveguides, Nat. Photonics 13, 697 (2019).

[15] X. S. Wang, Y. Su, and X. R. Wang, Topologically protected unidirectional edge spin waves and beam splitter, Phys. Rev. B 95, 014435 (2017).

[16] Y. Su, X. S. Wang, and X. R. Wang, Magnonic Weyl semimetal and chiral anomaly in pyrochlore ferromagnets, Phys. Rev. B 95, 224403 (2017).

[17] Y. Su and X. R. Wang, Chiral anomaly of Weyl magnons in stacked honeycomb ferromagnets, Phys. Rev. B 96, 104437 (2017).

[18] X. S. Wang, H. W. Zhang, and X. R. Wang, Topological Magnonics: A Paradigm for Spin-Wave Manipulation and Device Design, Phys. Rev. Appl. 9, 024029 (2018).

[19] W. A. Benalcazar, B. A. Bernevig, and T. L. Hughes, Quantized electric multipole insulators, Science 357, 61 (2017).

[20] J. Langbehn, Y. Peng, L. Trifunovic, F. von Oppen, and P. W. Brouwer, Reflection-Symmetric Second-Order Topological Insulators and Superconductors, Phys. Rev. Lett. 119, 246401 (2017).

[21] Z. Song, Z. Fang, and C. Fang, $(d-2)$-Dimensional Edge States of Rotation Symmetry Protected Topological States, Phys. Rev. Lett. 119, 246402 (2017).

[22] M. Ezawa, Higher-Order Topological Insulators and Semimetals on the Breathing Kagome and Pyrochlore Lattices, Phys. Rev. Lett. 120, 026801 (2018). 
[23] T. Liu, Y.-R. Zhang, Q. Ai, Z. Gong, K. Kawabata, M. Ueda, and F. Nori, Second-Order Topological Phases in NonHermitian Systems, Phys. Rev. Lett. 122, 076801 (2019).

[24] X.-W. Luo and C. Zhang, Higher-Order Topological Corner States Induced by Gain and Loss, Phys. Rev. Lett. 123, 073601 (2019).

[25] K. Kudo, T. Yoshida, and Y. Hatsugai, Higher-Order Topological Mott Insulators, Phys. Rev. Lett. 123, 196402 (2019).

[26] Z.-X. Li, Y. Cao, P. Yan, and X. R. Wang, Higher-order topological solitonic insulators, npj Comput. Mater. 5, 107 (2019).

[27] R. Chen, C.-Z. Chen, J.-H. Gao, B. Zhou, and D.-H. Xu, HigherOrder Topological Insulators in Quasicrystals, Phys. Rev. Lett. 124, 036803 (2020).

[28] J. Li, R.-L. Chu, J. K. Jain, and S.-Q. Shen, Topological Anderson Insulator, Phys. Rev. Lett. 102, 136806 (2009).

[29] C. W. Groth, M. Wimmer, A. R. Akhmerov, J. Tworzydło, and C. W. J. Beenakker, Theory of the Topological Anderson Insulator, Phys. Rev. Lett. 103, 196805 (2009).

[30] Y. Su, Y. Avishai, and X. R. Wang, Topological Anderson insulators in systems without time-reversal symmetry, Phys. Rev. B 93, 214206 (2016).

[31] S. Imhof, C. Berger, F. Bayer, J. Brehm, L. W. Molenkamp, T. Kiessling, F. Schindler, C. H. Lee, M. Greiter, T. Neupert, and R. Thomale, Topolectrical-circuit realization of topological corner modes, Nat. Phys. 14, 925 (2018).

[32] H. Araki, T. Mizoguchi, and Y. Hatsugai, Phase diagram of a disordered higher-order topological insulator: A machine learning study, Phys. Rev. B 99, 085406 (2019).

[33] Z. Su, Y. Kang, B. Zhang, Z. Zhang, and H. Jiang, Disorder induced phase transition in magnetic higher-order topological insulator: A machine learning study, Chin. Phys. B 28, 117301 (2019).

[34] A. Agarwala, V. Juričić, and B. Roy, Higher-order topological insulators in amorphous solids, Phys. Rev. Research 2, 012067(R) (2020).

[35] A. Szabo and B. Roy, Dirty higher-order Dirac semimetal: Quantum criticality and bulk-boundary correspondence, arXiv:2002.09475.

[36] B. A. Bernevig and T. L. Hughes, Topological Insulators and Topological Superconductors (Princeton University Press, Princeton, 2013).

[37] L. Trifunovic and P. W. Brouwer, Higher-Order Bulk-Boundary Correspondence for Topological Crystalline Phases, Phys. Rev. X 9, 011012 (2019).

[38] L. Fu and C. L. Kane, Topological insulators with inversion symmetry, Phys. Rev. B 76, 045302 (2007).

[39] J. C. Y. Teo, L. Fu, and C. L. Kane, Surface states and topological invariants in three-dimensional topological insulators: Application to $\mathrm{Bi}_{1-x} \mathrm{Sb}_{x}$, Phys. Rev. B 78, 045426 (2008).
[40] C.-K. Chiu, H. Yao, and S. Ryu, Classification of topological insulators and superconductors in the presence of reflection symmetry, Phys. Rev. B 88, 075142 (2013).

[41] T. Morimoto and A. Furusaki, Topological classification with additional symmetries from Clifford algebras, Phys. Rev. B 88, 125129 (2013)

[42] K. Shiozaki and M. Sato, Topology of crystalline insulators and superconductors, Phys. Rev. B 90, 165114 (2014).

[43] A. MacKinnon, The calculation of transport properties and density of states of disordered solids, Z. Phys. B 59, 385 (1985).

[44] A. Weiße, G. Wellein, A. Alvermann, and H. Fehske, The kernel polynomial method, Rev. Mod. Phys. 78, 275 (2006).

[45] C. W. Groth, M. Wimmer, A. R. Akhmerov, and X. Waintal, Kwant: A software package for quantum transport, New J. Phys. 16, 063065 (2014).

[46] P. Virtanen, R. Gommers, T. E. Oliphant, M. Haberland, T. Reddy, D. Cournapeau, E. Burovski, P. Peterson, W. Weckesser, J. Bright et al., SciPy 1.0: fundamental algorithms for scientific computing in Python, Nat. Methods 17, 261 (2020).

[47] C.-Z. Chen, J. Song, H. Jiang, Q.-F. Sun, Z. Wang, and X. C. Xie, Disorder and Metal-Insulator Transitions in Weyl Semimetals, Phys. Rev. Lett. 115, 246603 (2015).

[48] S. Liu, T. Ohtsuki, and R. Shindou, Effect of Disorder in a Three-Dimensional Layered Chern Insulator, Phys. Rev. Lett. 116, 066401 (2016).

[49] M. Janssen, Statistics and scaling in disordered mesoscopic electron systems, Phys. Rep. 295, 1 (1998).

[50] X. R. Wang, Y. Shapir, and M. Rubinstein, Analysis of multiscaling structure in diffusion-limited aggregation: A kinetic renormalization-group approach, Phys. Rev. A 39, 5974 (1989).

[51] C. Wang, Y. Su, Y. Avishai, Y. Meir, and X. R. Wang, Band of Critical States in Anderson Localization in a Strong Magnetic Field with Random Spin-Orbit Scattering, Phys. Rev. Lett. 114, 096803 (2015).

[52] J. H. Pixley, P. Goswami, and S. Das Sarma, Anderson Localization and the Quantum Phase Diagram of Three Dimensional Disordered Dirac Semimetals, Phys. Rev. Lett. 115, 076601 (2015).

[53] C. Wang, P. Yan, and X. R. Wang, Non-Wigner-Dyson level statistics and fractal wave function of disordered Weyl semimetals, Phys. Rev. B 99, 205140 (2019).

[54] T. Kawarabayashi, B. Kramer, and T. Ohtsuki, Anderson transitions in three-dimensional disordered systems with randomly varying magnetic flux, Phys. Rev. B 57, 11842 (1998).

[55] Y. Su, X. S. Wang, and X. R. Wang, A generic phase between disordered Weyl semimetal and diffusive metal, Sci. Rep. 7, 14382 (2017).

[56] W. H. Press, S. A. Teukolsky, W. T. Vetterling, and B. P. Flannery, Numerical Recipes in Fortran 77, 2nd ed. (Cambridge University Press, Cambridge, U.K., 1996). 